enten die richtige ist, ist insbesondere für die große Gruppe der StandardrisikoPatienten bislang weitgehend ungeklärt. Um sich diesem Thema zu nähern, benötigt man sehr große Zahlen von Patienten, die homogen therapiert werden. Der Postremissionstherapie(PRT)-Score der Studienallianz Leukämie [Pfirrmann $\mathrm{M}$ et al. Lancet Oncol. 2012;13(2):207-14] konnte zum ersten Mal klar Subgruppen von Patienten definieren, die von einer allogenen oder autologen Stammzelltransplantation besonders profitieren. Eine solche für die Therapieentscheidung bei AML-Patienten wichtige prädiktive
Information kann die hier vorgelegte Studie nicht liefern.

Zusammenfassend ist die $\mathrm{cnLOH}$ ein weiterer interessanter prognostischer Marker bei der AML. Bevor jedoch nun flächendeckend Mikroarrays bei der Erstdiagnose der AML eingesetzt werden sollten, sind weitere bestätigende Untersuchungen und die Evaluation der Methode im prädiktiven Setting notwendig. Zum tieferen Verständnis der AML als prognostisch heterogene Erkrankung taugen die Untersuchungen allemal und sollten uns insbesondere dazu aufrufen, die etablierten Prognosemarker bei diesen Patienten konsequent zu bestimmen, was unter den herrschenden ökonomischen Zwängen schon anspruchsvoll genug ist.



Prof. Dr. med. Markus Schaich Hämatologie, Onkologie und Palliativmedizin Rems-Murr-Klinikum Winnenden markus.schaich@remsmurr-kliniken.de

\title{
MRD-negativ bei AML? Prognose bei Älteren dennoch schlechter
}

Jüngere Patienten mit akuter myeloischer Leukämie (AML) können bei einem negativen Test auf eine minimale Resterkrankung nach der Ersttherapie zunächst von belastenden Therapien verschont werden. Ob dies auch für Ältere gilt, wurde in einer retrospektiven Studie überprüft.

A nhand der minimalen Resterkrankung (MRD, „minimal residual disease“) lässt sich auch bei Patienten mit AML die Prognose abschätzen und eine Entscheidung über das weitere therapeutische Vorgehen treffen. Bei jungen Patienten in der ersten kompletten Remission ist ein positiver MRD-Status Anlass, sich für eine allogene Stammzelltransplantation zu entscheiden. Bei negativem Testergebnis kann sie verschoben werden. In einer retrospektiven Studie wurde nun geprüft, ob sich Informationen zum MRD-Status, gemessen mittels Durchflusszytometrie, bei Älteren so zuverlässig nutzen lassen wie bei Jüngeren. Ausgewertet wurden Befunde von 210 AMLPatienten $(149<60$ Jahre alt). Sie hatten nach der Induktionstherapie eine morphologische komplette Remission. Als MRD-negativ galt der Nachweis von $<1$ Leukämiezelle/35.000 Leukozyten. Nach der Konsolidierung waren signifikant weniger Ältere MRD-negativ (11 vs. $28 \%$;

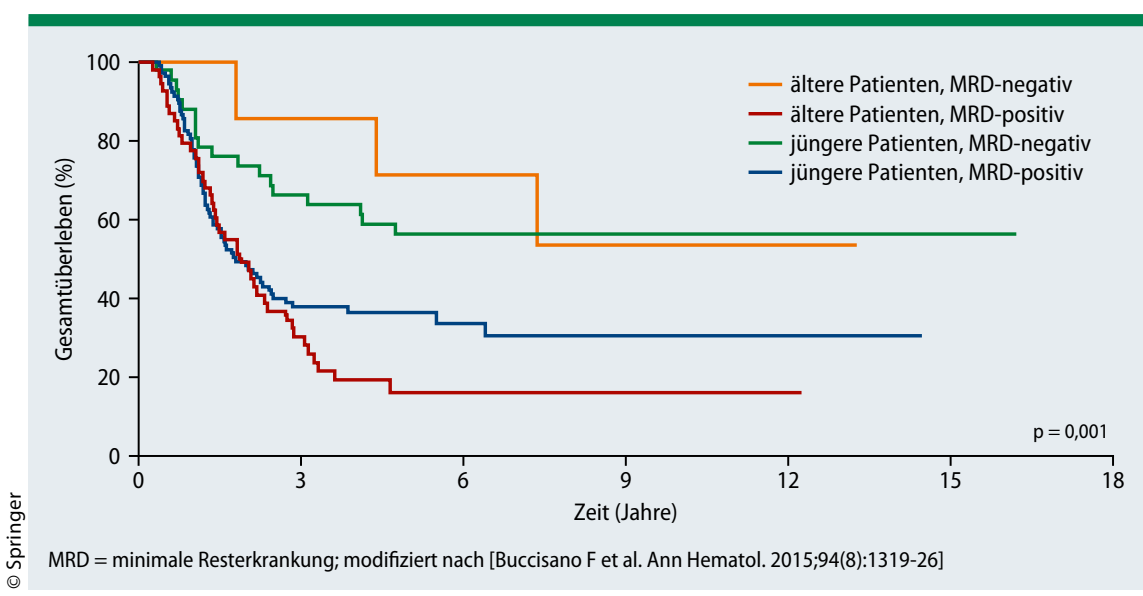

Abb. 1: Gesamtüberleben von Patienten mit akuter myeloischer Leukämie abhängig vom Alter und vom MRD-Status. $p=0,009)$. In beiden Altersgruppen war MRD-Negativität mit signifikant höheren progressionsfreien Überlebensraten nach 5 Jahren assoziiert ( $<60$ Jahre: 56 vs. $31 \%$; $>60$ Jahre: 57 vs. $13 \%$ ). Der Vorteil spiegelte sich auch in der Gesamtüberlebensrate (< 60 Jahre: 57 vs. $31 \%$; $>60$ Jahre: 71 vs. $16 \%$; Abb. 1). Bei Patienten mit positivem MRD-Test war die kumulative Inzidenz an Rückfällen (CIR) jeweils etwa verdoppelt (<60 Jahre: 59 vs. $24 \%$; > 60 Jahre: 83 vs. $42 \%)$. Jedoch war bei MRD-Negativität die CIR bei den Älteren nach 5 Jahren fast doppelt so hoch wie bei den Jüngeren (42 vs. $24 \%$ ). Die Forscher glauben, dass dieser Unterschied biologische Gründe hat und auf leukämischen Stammzellen beruht, die trotz der MRDNegativität die Leukämie wieder aufflammen lassen. Möglicherweise werden aber auch die Stammzellen durch den MRDTest nicht optimal erfasst.

Fazit: Ein negativer MRD-Test bei älteren AML-Patienten hat einen schlechteren Prognosewert als bei Jüngeren: Bei Patienten über 60 ist die CIR 5 Jahre nach Behandlungsbeginn doppelt so hoch. Eine Option könnte dann sein, die Therapie fortzusetzen und - altersgerecht intensiver zu gestalten, um das Gesamtüberleben zu verlängern. Peter Leiner

Buccisano F et al. Minimal residual disease negativity in elderly patients with acute myeloid leukemia may indicate different postremission strategies than in younger patients. Ann Hematol. 2015;94(8):1319-26. 